\title{
Thermal Conductivities of Unidirectional Materials
}

\author{
George S. Springer \\ Mechanical Engineering Department \\ University of Michigan \\ Ann Arbor, Michigan 48104 \\ StePhen $W$. TsaI \\ Department of Systems, Mechanical, and Aerospace Engineering \\ Washington University \\ St. Louis, Missouri 63130
}

\begin{abstract}
In this paper the composite thermal conductivities of unidirectional composites are studied and expressions are obtained for predicting these conductivities in the directions along and normal to the filaments. In the direction along the filament an expression is presented based on the assumption that the filaments and matrix are connected in parallel. In the direction normal to the filaments composite thermal conductivity values are obtained first by utilizing the analogy between the response of a unidirectional composite to longitudinal shear loading and to transverse heat transfer; second by replacing the filament-matrix composite with an idealized thermal model. The results of the shear loading analogy agree reasonably well with the results of the thermal model particularly at filament contents below about $60 \%$. These results were also compared to experimental data reported in the literature and good agreement was found between the data and those theoretical results that were derived for circular filaments arranged in a square packing array.
\end{abstract}

\section{INTRODUCTION}

Consiberable atTention has been given in recent years to the deter$\checkmark$ mination of the mechanical properties of composite materials. Relatively little information is available, however, on thermal properties, although they are of interest in such practical problems as heat transfer calculations and evaluating the thermoelastic behavior of materials. The goals in determining the thermal properties of composite materials are similar to those in mechanical analyses: given the thermal properties of each of the constituents, their proportion and geometrical relationships, find the thermal behavior of the composite system.

In this paper thermal conductivities of unidirectional composites will be discussed. The problem will be approached from two directions. First a numerical method will be presented based on the analogy between the response of the unidirectional composite to shear loading and to heat 


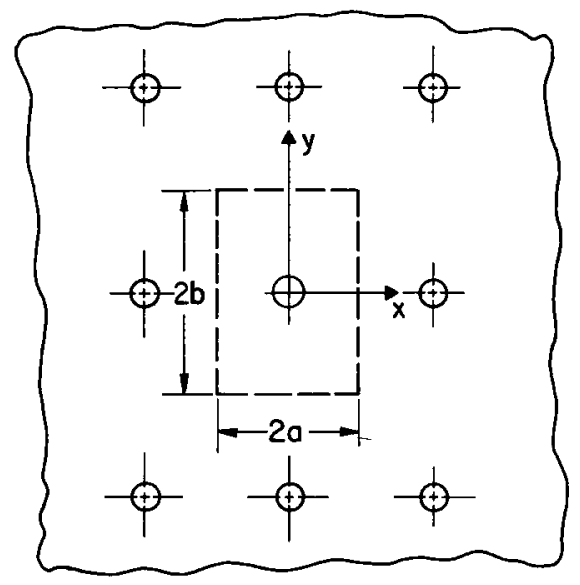

Figure 1. Arrangement of filaments, and the coordinate system used.

transfer. Second, a thermal model will be described, yielding approximate closed form results. This method is similar to Thornburg and Pears' [1] who recently obtained simple expressions for predicting thermal conductivities of filled and reinforced plastics. Their expressions were based on the assumption that the reinforcement (filament) and the matrix are arranged either in series or in parallel and that the resulting thermal model is analogous to series and parallel connected electrical circuits. Consequently the results are independent of the shape of the filaments and their geometrical arrangement. In the present analysis both of these effects will be taken into account.

\section{FORMULATION OF THE PROBLEM}

The problem of composite thermal conductivities of materials composed of unidirectional filaments embedded in a matrix will be considered here. In the analysis it will be assumed a.) that the composites are macroscopically homogenous, $b$.) that locally both the matrix and the filament are homogenous and isotropic, $\mathbf{c}$.) that the thermal contact resistance between the filament and the matrix is negligible, d.) that the problem is two dimensional, i.e. the temperature distribution is independent of $z$, (Figures $1 \& 2$ ), and e.) that the filaments are arranged in a rectangular periodic array (Figure 1). This last assumption implies that the filaments are equal and uniform in shape and size and are symmetrical about the $x, y$ axes. A rectangular packing array has been selected arbitrarily but this assumption is not essential. The results could be extended to other packing arrays provided that the symmetry conditions are satisfied. 


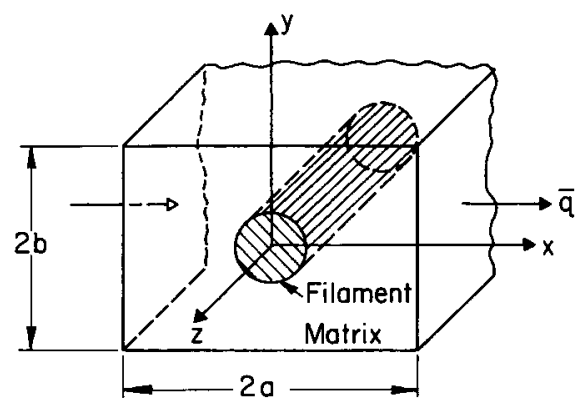

(a) Transverse heat transfers

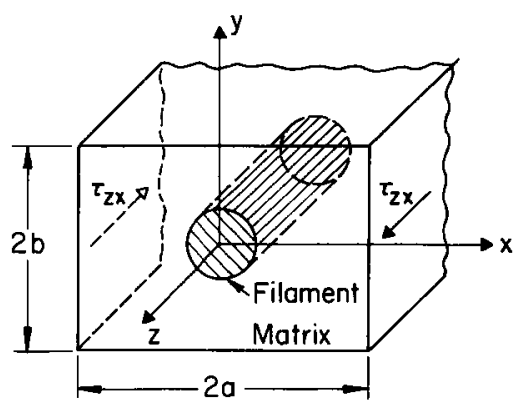

(b) Longitudinal shear loading

Figure 2. Fundamental element used in the analogy between transverse heat transfer and longitudinal shear loading.

Of primary importance are the composite thermal conductivities in the two principal directions, namely in the directions parallel $\left(k_{11}\right)$ and normal $\left(k_{22}\right)$ to the filaments. Once these conductivities are known, the conductivities in other directions can be calculated using the transformation properties given by Carlslaw and Jaeger [2]. The conductivity $k_{11}$ may be predicted by assuming that the filaments and the matrix are connected in parallel [1], i.e.

$$
\frac{k_{11}}{k_{m}}=\left(v_{f} k_{f} / k_{m}\right)+v_{m}
$$

where $v$ is the volume and $k$ the thermal conductivity; the subscripts $m$ and $f$ refer to the matrix and filament respectively. Similarly as in the determination of elastic constants of multiphase materials [3] this expression may be considered as an upper bound for $k$. This is borne out by a comparison between the theory (Eq. I) and experimental data as shown in reference [1]. In the remainder of the paper the composite thermal conductivity in the direction normal to the filaments $\left(k_{22}\right)$ will be investigated.

\section{SHEAR LOADING ANALOGY}

The temperature distribution at any local point in the element shown in Figure $2 a$ must satisfy the equation

$$
\frac{\partial^{2} T}{\partial x^{2}}+\frac{\partial^{2} T}{\partial y^{2}}=0
$$

Utilizing the symmetry conditions described previously the boundary condition corresponding to Eq. (2) may be written as 
Thermal Conductivities of Unidirectional Materials

$$
\begin{aligned}
& k \frac{\partial T}{\partial y}=0 \text { at } y=0 \text { and } y= \pm b \\
& T=T_{+a} \text { and } T=T_{-a} \text { at } x=+a \text { and } x=-a \\
& T=\frac{\Delta T}{2}=\text { const. at } x=0
\end{aligned}
$$

$k$ is the thermal conductivity of either the filament or the matrix, and $\Delta T$ is the temperature difference between the surfaces $x=a$ and $x=$ $-a\left(\Delta T=T_{+a}-T_{-a}\right)$. In addition to the boundary conditions given in Eq. (3) the conditions existing at the interface between the filament and the matrix must be specified. For zero thermal resistance at the interface

$$
\begin{gathered}
T_{f}=T_{m} \\
\left.k_{f} \frac{\partial T}{\partial n}\right|_{f}=\left.k_{m} \frac{\partial T}{\partial n}\right|_{m}
\end{gathered}
$$

Here $n$ symbolizes the direction normal to the interface.

One could now proceed to solve the above problem (Eqs. 2-4) by imposing a known uniform temperature distribution $(\Delta T)$ between the $x= \pm a$ surfaces. Having obtained a solution to this problem, one would first calculate the local heat transfer rate at points along the surface $x$ $=a$ and then compute an average heat transfer rate $\bar{q}$. Using this $\bar{q}$ value a composite thermal conductivity will be now defined as

$$
k_{22}=\frac{\bar{q}}{\Delta T / 2 a}
$$

Inspection of Eqs. (2-5) reveals an analogy between the above problem and that of longitudinal shear loading of a unidirectional composite. The latter problem is indicated in Figure $2 b$ and described in detail in ref. [4]. Equations (2-5) become identical to the equations and boundary conditions describing the longitudinal shear loading when the temperature $(T)$ is replaced by the displacement $(w)$, and the average heat transfer rate $(\bar{q})$ and the thermal conductivities $(k)$ are replaced by the average shear stress $\left(\bar{\tau}_{z x}\right)$ shear moduli $(G)$, respectively. Thus, the numerical solutions obtained by Adams and Doner [4] for the shear loading problem may be applied directly to the present problem by simply replacing the composite stiffness with the composite thermal conductivity and the shear modulus ratio with the thermal conductivity ratio.

\section{THERMAL MODEL}

The solution of the equations given in the previous section requires 


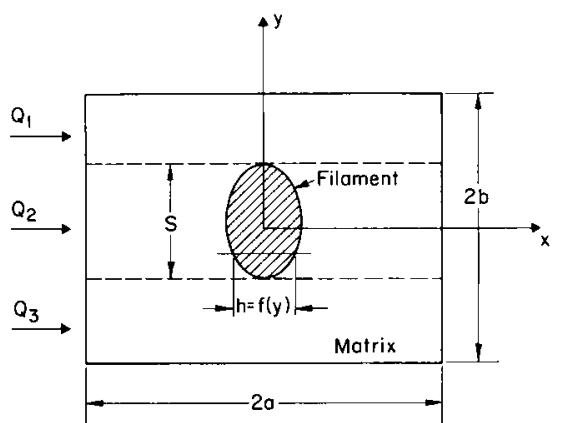

Figure 3. Fundamental element used in thermal modelling.

numerical techniques. Approximate closed form solutions for $k_{22}$ may be obtained by replacing the filament-matrix composite with an appropriately idealized thermal model. One of the simplest such models is that of the filament and the matrix arranged in series. The resulting composite thermal conductivity is [1]

$$
\frac{k_{22}}{k_{m}}=\frac{1}{\left(v_{f} k_{m} / k_{f}\right)+v_{m}}
$$

Again, as in the case of elastic constants [3] Eq. (6) may be considered a lower bound for $k$. The above expression is independent of both the filament shape and its geometrical arrangement. These factors may be included in the analysis by considering the thermal model shown in Figure 3. In this model it is assumed that the temperature difference is constant between $x= \pm a$ and that the total heat flow per unit length along the filament, $Q$, through the surface $x=-a$ may be divided into three independent parts, i.e. $Q=Q_{1}+Q_{2}+Q_{3}$. For this condition, and using the expression given for $k_{z 2}$ previously (Eq. 5) we may write

$$
k_{22} 2 b \cong 2 k_{m}\left(\frac{2 b-s}{2}\right)+\int_{\circ}^{s} \frac{2 a}{\frac{2 a-h}{k_{m}}+\frac{h}{k_{f}}} d y
$$

or

$$
\frac{k_{22}}{k_{m}} \cong\left(1-\frac{s}{2 b}\right)+\frac{a}{b} \int_{o}^{s} \frac{d y}{(2 a-h)+\left(h k_{m} / k_{f}\right)}
$$

The parameter $s$ is the maximum dimension of the filament in the $y$ direction and $h$ is the width of the filament at any given $y$ (Figure 3). While Eq. (8) is for a rectangular packing array, similar expressions could be obtained for other symmetrical packing arrays. In order to solve Eq. (8) the shape of the filament must be specified. For a square filament $(s=x=c o n s t)$ and square packing array $(a=b)$ Eq. (8) yields 
Thermal Conductivities of Unidirectional Materials

$$
\frac{k_{22}}{k_{m}}=\left(1-\sqrt{v_{f}}\right)+\frac{1}{\sqrt{I / v_{f}}+B / 2}
$$

For a cylindrical filament $(s=d)$ and square packing array Eq. (8) gives

$$
\begin{gathered}
\frac{k_{22}}{k_{m}}=\left(1-2 \sqrt{v_{f} / \pi}\right)+\frac{1}{B}\left[\pi-\frac{4}{\left.\sqrt{1-\left(B^{2} v_{f} / \pi\right.}\right)} \tan ^{-1} \frac{\left.\sqrt{1-\left(B^{2} v_{f} / \pi\right.}\right)}{1+\sqrt{B^{2} v_{f} / \pi}}\right] \\
B \equiv 2\left(\frac{k_{m}}{k_{f}}-1\right)
\end{gathered}
$$

\section{DISCUSSIONS}

Adams and Doner [4] present numerical results for the longitudinal shear loading problem for cylindrical, elliptical and square filaments and for selected volume ratios. ${ }^{*}$ Using these results one may now compare the composite thermal conductivities obtained from the shear loading analogy and from the thermal model equations (Eqs. 9, 10). Such a comparison, shown in Figure 4, indicates that the idealized thermal model

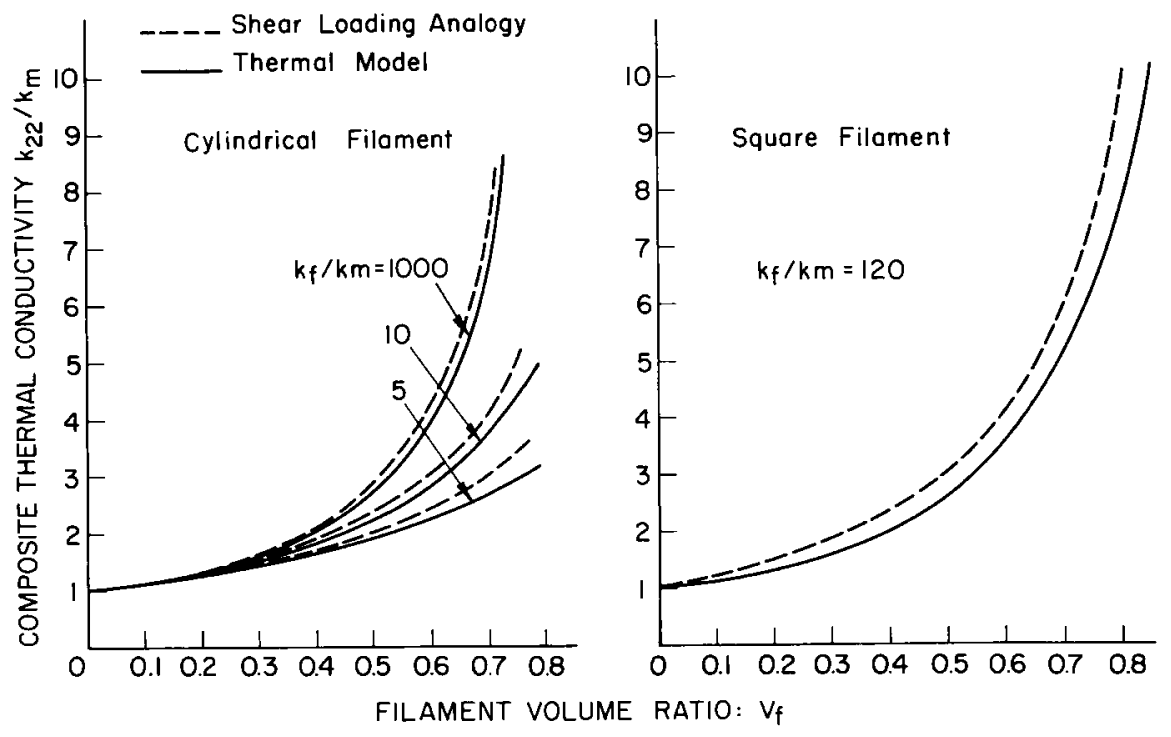

Figure 4. Comparison between the results of the shear loading analogy and a thermal model. Square packing array.

- A complete numerical analysis of the problem together with a FORTRAN IV computer program is given in reference [5]. 
gives a somewhat $(\sim 10 \%)$ lower thermal conductivity than the shear loading analogy, particularly at filament volume ratios above $\sim 75 \%$. Below filament volume ratios of about $60 \%$ the two methods agree within approximately $5 \%$ for cylindrical filaments.

In Figure 5 the theoretical results are compared with experimental data. The data shown are from reference [1] and are for various laminated glass-plastic, and graphite fabric-plastic composites. For a high thermal conductivity ratio $\left(k_{f} / k_{m}=666\right)$ the data agree reasonably well with the results of the shear model analogy but are higher than the values predicted by either one of the thermal models (Eqs. 6, 9, 10). The data also indicate that the assumption of cylindrical filaments is more appropriate than that of square filaments. Furthermore, it is interesting to note that the laminae-in-series model underestimates considerably both the data and the other theoretical results. At a lower thermal conductivity ratio $\left(k_{f} / k_{m} \cong 4.4\right)$ a similar trend is apparent, although from the four available data points definite conclusions cannot be reached.

Another interesting observation can be made from Figure 5 regarding the assumption of a square packing array. It is pointed out in reference [4] that a hexagonal packing array yields lower values for composite stiffness (and by analogy for composite thermal conductivity) than the square array for the same filament volume content. Thus, as is evi-

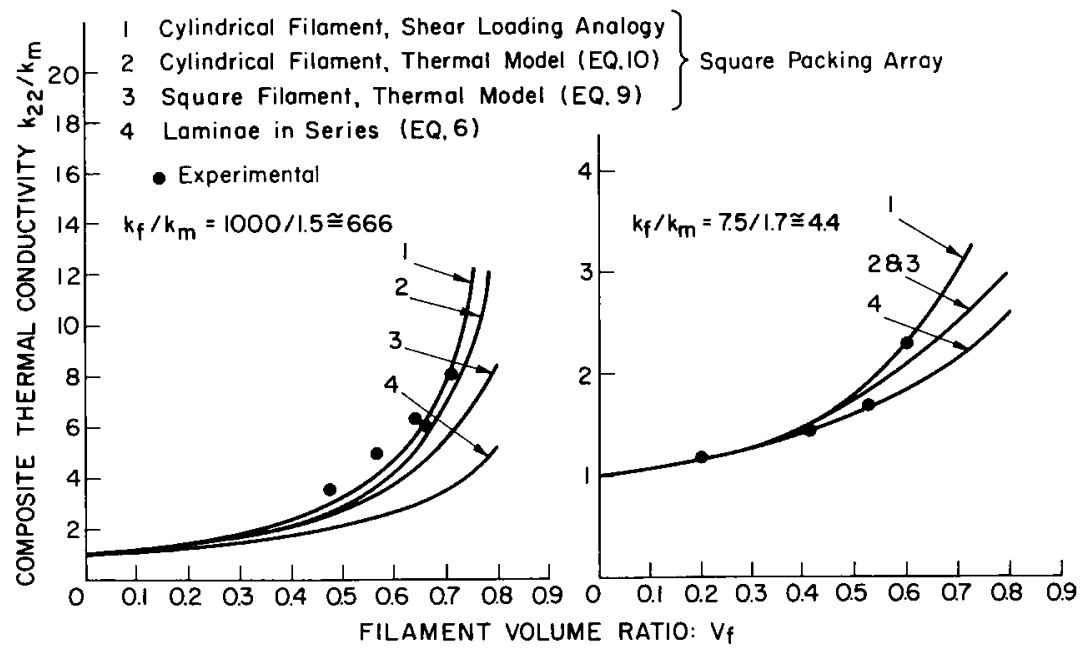

Figure 5. Comparison between experimental data and the results of the shear loading analogy and thermal models. Experimental data from Thornburg and Pears [1] for glass-plastic $\left(k_{f} / k_{m}=4.4\right)$ and graphite fabric-plastic $\left(k_{f} / k_{m}=666\right)$ composites are shown. 


\section{Thermal Conductivities of Unidirectional Materials}

dent from Figure 5 the square array gives values closer to the experimental data than would be given by the hexagonal array.

The foregoing results indicate that the composite thermal conductivity normal to the filaments may be predicted with reasonable accuracy by using either the analogy between heat transfer and shear loading or the thermal model given for cylindrical filaments in square packing arrays. It must be recognized of course that the composite thermal conductivity values thus obtained are subject to the approximations listed previously.

\section{NOMENCLATURE}



\section{REFERENCES}

1. J. D. Thornburg and C. D. Pears, "Prediction of the Thermal Conductivity of Filled and Reinforced Plastics," ASME Paper 65-WA/HT-4 (1965).

2. H. S. Carlslaw and J. C. Jaeger, Conduction of Heat in Solids, Oxford Press (1959), p. 38.

3. B. Paul, "Prediction of Elastic Constants in Multi-phase Materials," Trans AIME, Vol. 218 (1960), p. 36.

4. D. F. Adams and D. R. Doner, "Longitudinal Shear Loading of a Unidirectional Composite," J. Composite Materials, Vol. 1 (1967), p. 4.

5. S. W. Tsai, D. F. Adams and D. R. Doner, "Effect of Constituent Materials Properties on the Strength of Fiber Reinforced Composite Materials," Air Force Materials Laboratory Report AFML-TR-66-190, (August, 1966).

(received March 10, 1967) 